advertising these opportunities shortly, so look out for this if you are interested.

Psychiatry has the MRCPsych examination as the principal summative assessment of satisfactory completion of core specialist training. This, we believe, remains a reliable and essential test of the acquisition of the knowledge and competencies expected of a psychiatrist who is ready to progress to higher training. The current rating system for WPBAs in Assessments Online, however, does not sufficiently emphasise the essentially formative function of the process. As a consequence, many trainers have found it difficult to give robust and honest feedback and we have all become aware of the phenomenon of the trainee with a portfolio of perfect WPBA scores, baffled by their failure to pass the CASC exam. We are investigating ways of making the scoring system simpler and more aligned with judgements based on satisfactory development of competences in maintaining patient safety.

Workplace-based assessments, if used correctly, can be a powerful formative training tool. At the very least, they provide an opportunity for trainees to have their practice and competencies observed in a protected and structured manner. The challenge for trainers, the College and trainees themselves is to embrace the cultural training change that WPBAs represent so that they are used to support effective training. Workplace-based assessments are primarily a tool for helping an experienced clinician give robust and valid feedback to another clinician. To treat them as a tick-box exercise is to miss the point and lose their value. Those of us responsible for guiding members and trainees through the new training mechanisms have probably not been sufficiently clear or realistic about what is expected from trainers and trainees and there has certainly been a lack of clarity about the overwhelmingly formative function of WPBAs. For this we are sorry. We are learning too, and hope that the changes that we have outlined in this letter will move things forward. The College, too, must expect to receive robust and valid feedback about training initiatives, and we hope that colleagues will continue to survey trainer and trainee experiences and that we will be seen to act constructively and purposefully in response. We all want the highest possible quality training for psychiatrists and have to make the best use of the tools available.

1 Menon S, Winston M, Sullivan G. Workplace-based assessment: survey of psychiatric trainees in Wales. Psychiatr Bull 2009; 33: 468-74.

2 Babu KS, Htike MM, Cleak VE. Workplace-based assessments in Wessex: the first 6 months. Psychiatr Bull 2009; 33: 474-8.

3 Oyebode F. Competence or excellence? Invited commentary on... Workplace-based assessments in Wessex and Wales. Psychiatr Bull 2009; 33: 478-9.

Robert Howard, Dean, Royal College of Psychiatrists, email: robert.j.howard@kcl.ac.uk and Andrew Brittlebank, Associate Dean for Curriculum, Royal College of Psychiatrists, London

doi: $10.1192 / \mathrm{pb} .34 .3 .114 a$

\section{Medicalisation of stress belittles major mental illness}

Few would argue with Professor Kingdon when he states that 'Everybody gets stressed... . it's just the way we react that differs'.1 Indeed, as Kingdon asserts, there can be no doubt that continua exist between normality and certain states currently classified as mental disorders. However, the artificial dividing lines towards the ends of each spectrum, set purely by societal expectations, surely call into question the validity of those very diagnoses that have perpetuated the myth of massive unmet need in psychiatric services. ${ }^{2}$ Rather than adopting a stress model of diagnosis based on dimensions, perhaps diagnoses such as mild depression, social phobia and personality disorder should instead be dispensed with altogether.

On the other hand, major mental illness is not primarily stress-induced. Although environmental risk factors exist for schizophrenia, bipolar and unipolar (endogenous) mood disorders and dementia, there is no convincing evidence to suggest that these illnesses are any more likely than peptic ulcer, cancer or myocardial infarction to be triggered by psychosocial stress.

Furthermore, in psychiatric practice, a diagnosis is not a checklist of symptoms; it is a process we have each spent many years learning to craft. Symptoms and signs such as hallucinations and delusions undoubtedly sit on continua, but it does not follow that schizophrenia sits on a similar continuum. Using Kingdon's analogy, chest pain may vary in aetiology and sit on a continuum of frequency and severity, but myocardial infarction remains a categorical diagnosis.

Lastly, one should not reconceptualise and reclassify mental disorder as a response to the stigma attached to it. If cardiac illness were to suddenly become stigmatised, I doubt physicians would rewrite the diagnostic criteria for myocardial infarction. On the contrary, diagnosis would remain necessary for both immediate and long-term management, and it would still be vitally important to separate those with cardiopathy from those without.

1 Kingdon D. Everybody gets stressed . . . it's just the way we react that differs. Psychiatr Bull 2009; 33: 441-2.

2 Richman A, Barry A. More and more is less and less: the myth of massive psychiatric need. Br J Psychiatry 1985; 146: 164-8.

Richard Braithwaite, Specialist Registrar in Old Age Psychiatry, Portsmouth City Teaching Primary Care Trust, email: richard.braithwaite@ports.nhs.uk doi: 10.1192/pb.34.3.115

\section{Laughlin Prize winners: some further thoughts}

It seems entirely reasonable to argue that the number of e-letters (letters submitted online to the journal in response to an article) and/or e-responses (email responses to the corresponding author) an article receives is a proxy measure of the interest generated by the article and also the wider interest in the journal. Albeit lacking the robustness of the 'impact factor', why not call this the journal 'interest factor'? Although letters to the editor are way down the 'importance' hierarchy of academic publications, my letter on the Laughlin Prize ${ }^{1}$ still had six e-responses from trainees and four from the Laughlin Prize winners, hence my inference that The Psychiatrist probably has a high interest factor among its readers.

I give below an excerpt from an e-response I received from Professor McKeith, who won the Prize in 1981. I feel it is worth sharing because his eloquent, insightful and humble account answers three questions I set out to answer in my survey (to find out more about the winners, their preparation 